\title{
Combined CT angiography and SPECT myocardial perfusion imaging for the detection of functionally relevant coronary stenoses
}

\author{
JIAN-MING LI ${ }^{1}$, RONG-FANG SHI ${ }^{1}$, LI-REN ZHANG ${ }^{2}$, TING $^{1}{ }^{1}$ and ZHI DONG ${ }^{2}$ \\ Departments of ${ }^{1}$ Nuclear Medicine and ${ }^{2}$ Radiology, \\ Tianjin Medical University Cardiovascular Clinical Institute and TEDA \\ International Cardiovascular Hospital, Tianjin 300457, P.R. China
}

Received December 24, 2012; Accepted February 19, 2013

DOI: $10.3892 / \mathrm{mmr} .2013 .1354$

\begin{abstract}
The present study aimed to discuss and evaluate the diagnostic performances and incremental clinical values of combined computed tomography coronary angiography (CTCA) and single photon myocardial perfusion imaging (MPI) for the detection of functionally relevant coronary stenoses (FRCS). In total, 54 patients underwent CTCA, MPI and coronary angiography (CAG) within 30 days. The diagnostic performances of CTCA and combined CTCA plus MPI for the detection of significant coronary stenoses $(\geq 50 \%)$ were calculated on the patient- or vessel-based levels and included the indices of sensitivity (SN), specificity (SP), positive predictive value (PPV), negative predictive value (NPV) and accuracy (AC). On the patient-based level, the SP, PPV, NPV and AC of CTCA alone or combined with MPI in the detection of FRCS were 90.9 and 90.9, 71.9 and 96.9, 69.0 and 95.2, 92.0 and 93.9 and 79.6 and $94.4 \%$, respectively. On the vessel-based level, the SN, SP, PPV, NPV and AC of CTCA alone or combined with MPI in the detection of FRCS were 90.3 and 90.3, 87.6 and 98.9, 54.9 and $93.3,98.2$ and 98.4 and 88.0 and $97.7 \%$, respectively. The PPV, SP and AC of combined CTCA and MPI in the detection of FRCS were significantly improved compared with CTCA alone (all values, $\mathrm{P}<0.05$ ). The diagnostic performance of combined CTCA and MPI in the detection of FRCS not only retains a high SN and NPV, but also markedly improves SP, PPV and AC, which should play an incremental and significant role in the decision-making process for the revascularization therapy of coronary artery disease (CAD).
\end{abstract}

Correspondence to: Dr Jian-Ming Li, Department of Nuclear Medicine, Tianjin Medical University Cardiovascular Clinical Institute and TEDA International Cardiovascular Hospital, No. 61 Third Road, Tianjin Economic and Technological Development Area (TEDA), Tianjin 300457, P.R. China

E-mail: jianminglicn@126.com

Key words: myocardial perfusion imaging, SPECT, computed tomography, ischemia, coronary artery disease, functionally relevant coronary stenoses

\section{Introduction}

The latest advances in computed tomography (CT) make it possible to non-invasively and intuitively obtain the anatomical morphology of the coronary artery tree, particularly contributing to the identification of the magnitude, distribution and composition of coronary atherosclerosis $(1,2)$. It has been documented that CT coronary angiography (CTCA) has a high accuracy (AC) for the detection of obstructive coronary artery disease (CAD) compared with invasive coronary angiography (CAG) (2-5). CTCA reflects the anatomical morphology of the coronary arteries, without directly providing functional or prognostic information on CAD, that is to say it does not directly provide the pathophysiological significance correlated with the coronary lesions. As the extent and severity of ischemia are usually the key factors for deciding whether to select revascularization or medical therapy for $\mathrm{CAD}$, a non-invasive evaluation of the anatomical and functional information on the coronary lesions prior to performing CAG becomes a necessary and reasonable requirement. Myocardial perfusion imaging (MPI) using single photon emission CT (SPECT) is a well-established, non-invasive method that has been widely used for decades to provide functional information on coronary lesions. Numerous studies have proved that MPI is a cost-effective, non-invasive method for CAD management (6-8).

Combined CTCA and SPECT MPI should have positive incremental values and play a complementary role in CAD management by revealing the coronary anatomy and its relative functional significance, factors which are extremely important for the decision-making process and for improving the cost effectiveness with regard to CAD therapy. The present study aimed to discuss and evaluate the diagnostic performance and incremental clinical values of combined CTCA and MPI for the detection of functionally relevant coronary stenoses (FRCS).

\section{Materials and methods}

Study population. A total of 54 consecutive patients with suspected or known CAD were enrolled in the present study. 
All the cases underwent CTCA, MPI and CAG within 30 days. CAG was performed following CTCA and MPI, however, the sequence of CTCA and MPI depended on the individual clinical situation. The mean age of the study population was $57.5 \pm 9.4$ years and 36 patients were male $(66.7 \%)$. The exclusion criteria were as follows: unstable angina, acute coronary syndrome (ACS), frequently premature heartbeat, atrial fibrillation, contraindications for iodinated contrast agent, $\mathrm{X}$ syndrome, severe coronary calcification and motion artifacts affecting the measurement of the stenoses and patients with bypass grafts. All the patients provided written informed consent and the study was approved by the ethics committee of the Tianjin Medical University Cardiovascular Clinical Institute and TEDA International Cardiovascular Hospital, Tianjin, China.

\section{CTCA}

Image acquisition. All the scans were performed on a 64-slice CT scanner (GE Light Speed VCT, GE Healthcare, Amersham, UK). The patients were required to fast for $4 \mathrm{~h}$ prior to the CT imaging. The patients with pre-scan heart rates of $>65$ b.p.m were administered $\beta$-blockers (Betaloc tablets, 25-100 mg). Prior to starting the scans, all the patients were required to take $0.5 \mathrm{mg}$ nitroglycerin sublingually. The acquisition parameters included a rotation time of $350 \mathrm{msec}$, a tube voltage of $120 \mathrm{kV}$, a tube current of $650-800 \mathrm{~mA}$ and a collimation of $0.6 \mathrm{~mm}$. The scan scope ranged from the carina of the trachea to $2 \mathrm{~cm}$ below the diaphragm. The total scan time was $\sim 12 \mathrm{sec}$. Briefly, for the pre-scan, a bolus of $20 \mathrm{ml}$ iohexol contrast agent $(350 \mathrm{mg} /$ $\mathrm{ml}$ ) was delivered intravenously through the antecubital vein at a flow rate of $4 \mathrm{ml} / \mathrm{sec}$ using a high pressure injector. Bolus tracking techniques were performed with a region of interest placed in the ascending aorta in order to record the peak time of enhancement and confirm the delay time for starting the acquisition. A bolus of 70-80 $\mathrm{ml}$ of iohexol was then intravenously injected into the bloodstream at a speed of 4-5 ml/ sec through the antecubital vein. The patients were required to hold their breath for the duration of the scan.

Image reconstruction and interpretation. The acquisition data were transferred to a GE Advanced Workstation (GE Healthcare) and a late diastole phase (75\% R-R time) was automatically reconstructed. If the quality of the reconstructed images was not satisfactory, then images of a $45-85 \%$ R-R time were reconstructed and reviewed in order to select the best reconstructed images, i.e., those which clearly depicted the coronary arteries. The images were visualized on the screen in the form of multiple planar reformations (MPRs), with virtual rendering volume (VRT) and maximal intensity projection (MIP). The images were visually and independently evaluated by two experienced readers (with $>3$ years of experience in cardiovascular imaging), who were blinded to the results of the MPI. The final diagnosis was reached when a consensus was agreed by the two readers. The coronary arteries were subdivided according to the 15 -segment model proposed by the American Heart Association (9). Each segment was visually assessed and reported as a $\geq 50$ or $<50 \%$ narrowing and allocated to the left main (LM), left anterior descending (LAD), left circumflex (LCX) and right coronary arteries (RCA). The narrowest lesion was regarded as the final diagnosis for diffuse or multiple stenoses in a single vessel.

\section{MPI}

Imaging acquisition. Exercise stress/rest-gated MPIs were performed on all the patients prior and subsequent to CTCA. $\beta$-blockers, calcium channel blockers and nitrates were discontinued for $\geq 24 \mathrm{~h}$ prior to MPI. The exercise stress tests were performed according to the modified Bruce protocol on a bicycle ergometer with a 12-lead ECG. Blood pressure measurements were taken at the baseline and then every 2 min during the procedure. The endpoints for the stress tests included any of the following indices: reaching the target heart beats [(220 - age in years) x 85\%], ischemic ST-segment horizontal or downslope depression of $\geq 2 \mathrm{~mm}$, the emergence of typical angina, severe cardiac arrhythmia, hypertension $(\geq 240 / 120 \mathrm{mmHg}$ ) or a fall in systolic pressure by $\geq 40 \mathrm{mmHg}$. At the peak of exercise, a 925-MBq dose of technetium-99m-labelled methoxyisobutyl isonitrile $\left({ }^{99 \mathrm{~m}} \mathrm{Tc}-\mathrm{MIBI}\right)$ was injected into the bloodstream through a vein and the patient continued to pedal for an additional $1 \mathrm{~min}$. The ECG and blood pressure were monitored prior to, throughout and subsequent to the injection. The acquisition for the stress-gated SPECT study was performed $1 \mathrm{~h}$ subsequent to the injection. The rest studies began acquisition $1.5 \mathrm{~h}$ subsequent to an injection using the same doses. The GE Millenium VG and Hawkeys dual-detector SPECT imaging system (GE Healthcare) was used. The acquisition parameters were as follows: a low-energy, high-resolution collimator; a $20 \%$ symmetric window at $140 \mathrm{keV}$; a $64 \times 64$ matrix; an elliptical orbit with step-and-shoot acquisition at $6^{\circ}$ intervals over $180^{\circ}$ from the right anterior oblique (RAO) $45^{\circ}$ angle to the left posterior oblique (LPO) $45^{\circ}$ angle; and a $25 \mathrm{sec}$ dwell time per stop. The acquisitions were gated at 8 frames per R-R cycle with a $50 \%$ window of the accepted heart rate.

Image reconstruction and interpretation. All the data were transferred to an eNTEGRA workstation (GE Healthcare) and reconstructed using an iterative reconstruction algorithm ( 2 iterations and 10 subsets; Butterworth pre-filtering function, a gradient order of 5.0 and a frequent cut-off of 0.25 ) without $\mathrm{x}$-ray attenuation correction. The images were reconstructed into short, horizontal and vertical long axial sections. At the same time, polar maps of wall motion and wall thickening were obtained by a special software package (ECToolbox and Multi-dim).

SPECT image interpretations were visually performed, with a consensus agreed by two experienced nuclear physicians; the readers were unaware of the CTCA results. The tomographic slices were divided into 17 segments according to the American Heart Association model. The MPI results were divided into two categories: normal perfusion, which was defined as homogenous radioactive distributions in the myocardium and no defective segments; and perfusion defects, including reversible and fixed perfusion defects. The reversible pattern showed that the localized segments of decreased perfusion observed during stress were no longer apparent or that they demonstrated a partial improvement at rest. The fixed pattern showed that the localized segments of decreased perfusion during stress were unchanged between the stress and rest images. The reversible perfusion defects were considered to represent myocardial ischemia and the fixed perfusion defects with concurrent regional wall motion abnormalities were considered to be myocardial scars. The perfusion defects were allocated to coronary artery territories as previously 
Table I. Clinical characteristics of patients referred for invasive angiography.

\begin{tabular}{lc}
\hline Parameters & Overall $(\mathrm{n}=54)$ \\
\hline Gender, $\mathrm{n}(\mathrm{M} / \mathrm{F})$ & $36 / 18$ \\
Age, mean $\pm \mathrm{SD}$ (years) & $57.5 \pm 9.4$ \\
Body mass index, mean $\pm \mathrm{SD}\left(\mathrm{kg} / \mathrm{m}^{2}\right)$ & $26.6 \pm 3.0$ \\
Risk factors for CAD & \\
Diabetes mellitus, $\mathrm{n}(\%)$ & $3(12)$ \\
Hypertension, $\mathrm{n}(\%)$ & $33(61)$ \\
Hypercholesterolemia, $\mathrm{n}(\%)$ & $12(22)$ \\
Positive family history, $\mathrm{n}(\%)$ & $8(15)$ \\
History of smoking, $\mathrm{n}(\%)$ & $22(41)$ \\
Symptoms & \\
Aymptomatic, $\mathrm{n}(\%)$ & $3(6)$ \\
Dyspnea, $\mathrm{n}(\%)$ & $22(41)$ \\
Non-anginal chest pain, $\mathrm{n}(\%)$ & $5(9)$ \\
Atypical angina pectoris, $\mathrm{n}(\%)$ & $12(22)$ \\
Typical angina pectoris, $\mathrm{n}(\%)$ & $13(24)$ \\
\hline
\end{tabular}

CAD, coronary artery disease.

repoted (10). The defects in the anterior and septal walls were allocated to the LAD and defects in the lateral wall were allocated to the RCA. The defects affecting the LAD and LCX regions together were considered as LM lesions. In the watershed region, allocation was determined according to the main extension of the defect onto the lateral, anterior or inferior wall.

$C A G$. Conventional CAG was performed according to the standard Judkins catheterization technique. Multiple views of each coronary artery were obtained and digitally stored on a designated workstation following intracoronary application of an iodinated contrast agent. The angiograms were observed and evaluated by two experienced interventional cardiologists who were blinded to the results of the CTCA and MPI. The final diagnosis was reached by consensus between the two readers. A visual assessment of a vessel narrowing of $\geq 50 \%$ was defined as significant stenosis.

Data analysis and statistics. FRCS was defined as a vessel narrowing of $\geq 50 \%$ on CAG with a corresponding perfusion defect on MPI (10). The performance indices of CTCA for the detection of significant stenosis on the patient- or vessel-based level were calculated using $\mathrm{CAG}$ as a reference. The indices of CTCA alone or combined with MPI in the detection of FRCS on the patient- or vessel-based level were also calculated using CAG plus MPI as a reference. The gold standard defines FRCS as a $\geq 50 \%$ stenosis on CAG with a corresponding perfusion defect on MPI in the same vessel territory. A true positive (TP) CTCA or combined CTCA and MPI segment was defined according to the gold standard. Comparisons of the statistical differences between groups were performed using a Pearson Chi-square test or a Fisher's exact probability test. $\mathrm{P}<0.05$ was considered to indicate a statistically significant difference.
Table II. Diagnostic indices of CTCA for the detection of $\geq 50 \%$ coronary narrowing in 216 segments of 54 patients.

\begin{tabular}{lcc}
\hline Indices & $\begin{array}{c}\text { Patient-based level } \\
(\mathrm{n}=54)\end{array}$ & $\begin{array}{c}\text { Vessel-based level } \\
(\mathrm{n}=216)\end{array}$ \\
\hline True positive, $\mathrm{n}$ & 28 & 51 \\
True negative, $\mathrm{n}$ & 21 & 161 \\
False positive, $\mathrm{n}$ & 1 & 2 \\
False negative, $\mathrm{n}$ & 4 & 2 \\
SN, \% & 87.5 & 96.2 \\
SP, \% & 95.5 & 98.8 \\
PPV, \% & 96.6 & 96.2 \\
NPV, \% & 84.0 & 98.8 \\
AC, \% & 90.7 & 98.2 \\
\hline
\end{tabular}

CTCA, computed tomography coronary angiography; SN, sensitivity; SP, specificity; PPV, positive predictive value; NPV, negative predictive value; $\mathrm{AC}$, accuracy.

The data statistics were automatically completed using the SPSS 13.0 statistical software.

\section{Results}

Patient characteristics. CTCA, MPI and CAG were performed without complications in all patients. The patient characteristics are described in detail in Table I.

CTCA versus CAG. With CAG regarded as the reference, the performance indices of CTCA for detecting significant stenoses on the patient- or vessel-based level are listed in Table II. The data showed that CTCA had a high sensitivity (SN), specificity (SP) and AC for the detection of obstructive CAD compared with CAG.

CTCA alone or combined with MPI compared with CAG plus MPI. According to the predefined reference standard, the performance indices of CTCA alone or combined with MPI are listed in Table III and the comparisons between them analyzed. Whether on the patient- or vessel-based level, the positive predictive value (PPV), SP and AC values of the combined CTCA and MPI for the detection of FRCS were significantly improved compared with CTCA alone, but the SN and negative predictive value (NPV) did not change greatly.

Fig. 1 shows a typical case of a 51-year-old male with angina pectoris. The anatomical and physiological information provided by CTCA and MPI showed that interventional therapy should be directed at the lesions in the LAD and RCA. The patient consequently received successful stent therapy for these lesions.

According to the predefined standard, 31 vessels with FRCS were detected among 54 patients. A total of 7 patients with three-vessel artery stenoses were included in the present study, 6 of which were unbalanced three-vessel stenoses, with 18 vessels narrowed by $\geq 50 \%$ among the total 24 vessels. Only 5 vessels with FRCS were identified by CAG plus MPI in 6 of 
Table III. Diagnostic indices and comparisons of CTCA alone or combined with MPI in 216 segments of 54 patients.

\begin{tabular}{|c|c|c|c|c|c|c|}
\hline \multirow[b]{2}{*}{ Indices } & \multicolumn{2}{|c|}{ Patient-based level $(n=54)$} & \multirow[b]{2}{*}{ P-values, $\mathrm{n}\left(\chi^{2}\right)$} & \multicolumn{2}{|c|}{ Vessel-based level ( $\mathrm{n}=216)$} & \multirow[b]{2}{*}{ P-values, $\mathrm{n}\left(\chi^{2}\right)$} \\
\hline & CTCA (\%) & CTCA+MPI (\%) & & CTCA (\%) & CTCA+MPI (\%) & \\
\hline True positive & 20 & 20 & & 28 & 28 & \\
\hline True negative & 23 & 31 & & 162 & 183 & \\
\hline False positive & 9 & 1 & & 23 & 2 & \\
\hline False negative & 2 & 2 & & 3 & 3 & \\
\hline $\mathrm{SN}$ & 90.9 & 90.9 & $1.00^{\mathrm{a}}$ & 90.3 & 90.3 & $1.00^{\mathrm{a}}$ \\
\hline SP & 71.9 & 96.9 & $0.01^{\mathrm{a}}$ & 87.6 & 98.9 & $0.00^{\mathrm{b}}(18.92)$ \\
\hline PPV & 69.0 & 95.2 & $0.03^{\mathrm{a}}$ & 54.9 & 93.3 & $0.00^{\mathrm{b}}(13.07)$ \\
\hline NPV & 92.0 & 93.9 & $1.00^{\mathrm{a}}$ & 98.2 & 98.4 & $1.00^{\mathrm{a}}$ \\
\hline $\mathrm{AC}$ & 79.6 & 94.4 & $0.02^{\mathrm{b}}(5.3)$ & 88.0 & 97.7 & $0.00^{\mathrm{b}}(15.33)$ \\
\hline
\end{tabular}

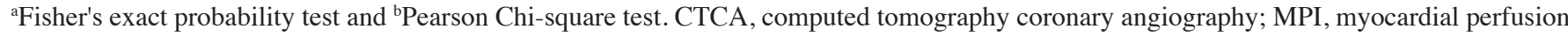
imaging; SN, sensitivity; SP, specificity; PPV, positive predictive value; NPV, negative predictive value; AC, accuracy.
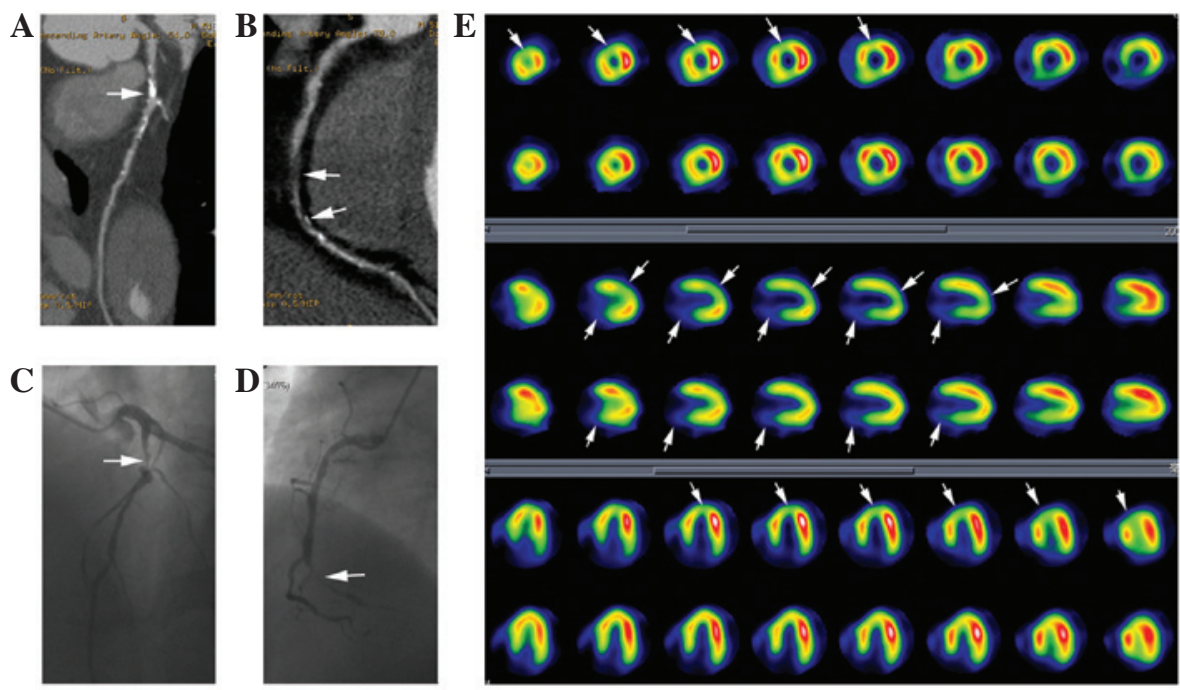

Figure 1. Patient with stenosis of the LAD artery classified as having $\geq 50 \%$ stenosis and RCA classified as nearly occluded on CTCA, associated with reversible perfusion defects in the anterior wall and partial apex and fixed perfusion defects in the posterior wall. (A) Multiple planar reformation (MPR) on CTCA shows the proximal and middle segment of the LAD artery with partially calcified, narrowed lumen (arrow). (B) MPR on CTCA shows the whole course of RCA with multiple, non-calcified, narrowed lumen and near occlusion in the middle segment of the RCA (arrows). (C) CAG of LAD shows severe stenosis (arrow), which corresponded to the CTCA results. (D) CAG of RCA shows near occlusion (arrow), which is also consistent with the CTCA results. (E) SPECT MPI study during stress (odd rows) and rest (even rows) shows reversible perfusion defects (arrows) in the anterior wall and partial apex corresponding to the territory of the LAD artery and fixed perfusion defects (arrows) in the posterior wall corresponding to the territory of the RCA. LAD, left anterior descending; RCA, right coronary artery; CTCA, computed tomography coronary angiograhy; CAG, coronary angiography; SPECT MPI, single photon emission CT myocardial perfusion imaging.

the patients with unbalanced three-vessel stenoses, which inferred that even more vessels with FRCS were missed due to negative MPIs. One patient with a balanced three-vessel stenosis was included in the present study whose MPI result was negative.

\section{Discussion}

The most significant results from the present study are as follows: First, CTCA had a high accuracy for the detection of significant coronary artery stenoses, as shown in previous studies (11-13). Second, in the detection of FRCS, CTCA alone had a relatively low SP, PPV and AC. Third, in the detection of FRCS, combining CTCA and SPECT MPI significantly improved the SP and PPV and maintained the SN and NPV when compared with CTCA alone.

In recent years, with the marked development of CT techniques, particularly the emergence of multi-slice CT (MSCT), which has a faster gantry rotation, multi-detector array and dual-source system, it is possible to overcome the motion artifacts of the heart and non-invasively create morphological images of the coronary arteries, usually identified by CTCA. CTCA is able to directly reveal the site and severity of a coronary lesion. In addition, the length of the stenosis and the distribution, magnitude and even the composition of the plaque may be precisely revealed and classified (calcified versus 
non-calcified) $(2,3,12,14)$. CTCA does not directly provide the hemodynamic significance correlated with the abnormalities of the coronary arteries, which would be extremely important to the therapeutic strategies for CAD. The main limitations for considering coronary narrowing only are as follows: First, the severity of the stenosis is only a modest surrogate of coronary resistance that does not include other lesion characteristics, including the length of the narrowing, the shape and eccentricity of the plaque and serial stenosis. These factors may also greatly impede the blood perfusion to the myocardium. Second, the vasomotor tone and coronary collateral flow, which are known to affect myocardial perfusion, may not be assessed by a simple measurement of stenosis severity (15). Therefore, functional imaging is required in order to reveal the pathophysiological changes correlated with the coronary lesions.

MPI is a well-established and documented non-invasive cardiac imaging method used for the diagnosis, prognosis and risk stratification of CAD, which has been practiced in the clinic for decades. Numerous results from evidence-based medicinal studies have confirmed that MPI is extremely effective, possessing guided significance and a better cost-benefit ratio for patient management $(6,8,11,16)$. A normal myocardial perfusion scan study indicated that patients were at a low risk for subsequent cardiac events. Therefore, no interventional therapy is usually required, but risk increases exponentially with worsening perfusion abnormality $(17,18)$. Numerous study results have led to a large number of class I indications for MPS in the risk assessment of patients with an intermediate or high likelihood of CAD $(8,11)$.

CTCA and MPI provide differing information for CAD from respective angles, thus it is difficult to directly compare CTCA with MPI as they show different things. The correlation between CTCA and MPI is supplemental rather than substitutional. For example, a positive MPI plus significant coronary stenoses are confident indicators for revascularization. According to the American Heart Association guidelines for cardiac intervention therapy and radionuclide imaging, a non-invasive morphological and functional method should be used prior to revascularization in patients with chronic stable angina. The guidelines also state that coronary stenosis with corresponding ischemia is one of the major indications for performing revascularization and that conservative therapy should be adopted where there is no significant stenoses or corresponding ischemia $(8,11,19)$. These guidelines aid in making the correct decision to ensure that patients benefit from treatments when non-invasively obtaining the functional and anatomical information on the coronary lesions. Of note is the fact that for patients with a high pre-test likelihood of CAD (>85\%), a negative MPI study does not exclude a diagnosis of coronary atherosclerosis. For example, in the present study, only 5 vessels with FRCS were identified by CAG plus MPI in 6 patients with unbalanced three-vessel stenoses, while MPI was negative for one patient with a balanced three-vessel stenosis. The reasons for this include the fact that the diagnostic criteria of MPI are classified as normal or abnormal depending on the relative distribution of the radiopharmaceuticals in the myocardium. MPI may just detect the abnormal territories corresponding to one or two relatively severe coronary artery stenoses in the patients with three-vessel disease. MPI may even be negative for the balanced reduction of perfusion to the myocardium in patients with balanced three-vessel stenoses. Therefore, MPI may miss certain patients with balanced three-vessel disease, whereas CTCA is unlikely to miss severe or extensive coronary atherosclerosis. It may be inferred that for patients with three-vessel disease, the $\mathrm{SN}$ for the detection of FRCS is lower due to negative MPI results, however, the extensive and diffuse lesions of the multiple coronary arteries presented on the CTCA remain highly indicative of the patients with a high risk. To avoid these potential clinical limitations, CTCA combined with MPI is required and is valuable for obtaining a deeper knowledge of CAD.

The incremental values that guide therapeutic strategies using combined CTCA and MPI for the detection of FRCS are summarized as follows: A patient without significant coronary stenoses or perfusion defects should undergo primary prevention and control of risk factors; a patient with significant coronary stenoses and without corresponding perfusion defects should turn to aggressive medical therapy and control of risk factors; and a patient with significant coronary stenoses and corresponding perfusion defects should actively undergo revascularization in order to obtain an improved prognosis rather than undergoing conservative therapy $(18,20)$. To evaluate the diagnostic performance of CTCA combined with MPI for the detection of FRCS, the use of MPI is the accepted reference standard, which has been previously used for the evaluation of the clinical values of hybrid heart imaging devices in patients with known or suspected CAD $(21,22)$.

Although CTCA alone has a high accuracy for the detection of obstructive CAD and an excellent NPV for ruling out FRCS, its PPV and SP remain relatively low. Using combined CTCA and MPI may markedly increase the diagnostic performance for the detection of FRCS when compared with CTCA alone, which may provide comprehensive information and play a significant role in the decision-making process for $\mathrm{CAD}$ management.

\section{Acknowledgements}

This study was supported by grants from the Binhai Health Bureau Medicine Health Science and Technology Project (2011BHKY004) and the Tianjin Health Bureau Technology Fund (2011KZ12).

\section{References}

1. Ropers D, Baum U, Pohle K, et al: Detection of coronary artery stenoses with thin-slice multi-detector row spiral computed tomography and multiplanar reconstruction. Circulation 107: 664-666, 2003

2. Hoffmann MH, Shi H, Schmitz BL, et al: Noninvasive coronary angiography with multislice computed tomography. JAMA 293: 2471-2478, 2005

3. MolletNR, CademartiriF, van Mieghem CA, et al:High-resolution spiral computed tomography coronary angiography in patients referred for diagnostic conventional coronary angiography. Circulation 112: 2318-2323, 2005.

4. Leber AW, Knez A, von Ziegler F, et al: Quantification of obstructive and nonobstructive coronary lesions by 64-slice computed tomography: a comparative study with quantitative coronary angiography and intravascular ultrasound. J Am Coll Cardiol 46: 147-154, 2005.

5. Maffei E, Palumbo A, Martini C, et al: Diagnostic accuracy of 64-slice computed tomography coronary angiography in a large population of patients without revascularization: registry data and review of multicentre trials. Radiol Med 115: 368-384, 2010. 
6. Underwood SR, Anagnostopoulos C, Cerqueira M, et al; British Cardiac Society; British Nuclear Cardiology Society; British Nuclear Medicine Society; Royal College of Physicians of London; Royal College of Radiologists: Myocardial perfusion scintigraphy: the evidence. Eur J Nucl Med Mol Imaging 31: 261-291, 2004.

7. Go V, Bhatt MR and Hendel RC: The diagnostic and prognostic value of ECG-gated SPECT myocardial perfusion imaging. J Nucl Med 45: 912-921, 2004.

8. Klocke FJ, Baird MG, Lorell BH, et al; American College of Cardiology; American Heart Association Task Force on Practice Guidelines; American Society for Nuclear Cardiology: ACC/ AHA/ASNC guidelines for the clinical use of cardiac radionuclide imaging - executive summary. A report of the American College of Cardiology/American Heart Association Task Force on Practice Guidelines (ACC/AHA/ASNC Committee to Revise the 1995 Guidelines for the Clinical Use of Cardiac Radionuclide (Imaging). Circulation 108: 1404-1418, 2003.

9. Austen WG, Edwards JE, Frye RL, et al: A reporting system on patients evaluated for coronary artery disease. Report of the Ad Hoc Committee for Grading of Coronary Artery Disease, Council on Cardiovascular Surgery, American Heart Association. Circulation 51 (Suppl 4): 5-40, 1975.

10. Gaemperli O, Schepis T, Valenta I, et al: Functionally relevant coronary artery disease: comparison of 64-section CT angiography with myocardial perfusion SPECT. Radiology 248: 414-423, 2008

11. Hendel RC, Patel MR, Kramer CM, et al; American College of Cardiology Foundation Quality Strategic Directions Committee Appropriateness Criteria Working Group; American College of Radiology; Society of Cardiovascular Computed Tomography; Society for Cardiovascular Magnetic Resonance; American Society of Nuclear Cardiology; North American Society for Cardiac Imaging; Society for Cardiovascular Angiography and Interventions; Society of Interventional Radiology: ACCF/ACR/ SCCT/SCMR/ASNC/NASCI/SCAI/SIR 2006 appropriateness criteria for cardiac computed tomography and cardiac magnetic resonance imaging: a report of the American College of Cardiology Foundation Quality Strategic Directions Committee Appropriateness Criteria Working Group, American College of Radiology, Society of Cardiovascular Computed Tomography, Society for Cardiovascular Magnetic Resonance, American Society of Nuclear Cardiology, North American Society for Cardiac Imaging, Society for Cardiovascular Angiography and Interventions, and Society of Interventional Radiology. J Am Coll Cardiol 48: 1475-1497, 2006.

12. Sato A, Hiroe M, Tamura M, et al: Quantitative measures of coronary stenosis severity by 64-Slice CT angiography and relation to physiologic significance of perfusion in nonobese patients: comparison with stress myocardial perfusion imaging. J Nucl Med 49: 564-572, 2008.

13. Wijns W: Anatomic-functional imaging by single-photon emission computed tomography/computed tomography as the corner stone of diagnosis and treatment for coronary patients: a glimpse into the (near) future? J Am Coll Cardiol 49: 1068-1070, 2007.
14. Cyrus T, Gropler RJ and Woodard PK: Coronary CT angiography (CCTA) and advances in CT plaque imaging. J Nucl Cardiol 16: 466-473, 2009.

15. Dorbala S, Hachamovitch R and Di Carli MF: Myocardial perfusion imaging and multidetector computed tomographic coronary angiography: appropriate for all patients with suspected coronary artery disease? J Am Coll Cardiol 48: 2515-2517, 2006

16. Hachamovitch R, Hayes SW, Friedman JD, Cohen I and Berman DS: Stress myocardial perfusion single-photon emission computed tomography is clinically effective and cost effective in risk stratification of patients with a high likelihood of coronary artery disease (CAD) but no known CAD. J Am Coll Cardiol 43 : 200-208, 2004

17. Berman DS, Abidov A, Kang X, et al: Prognostic validation of a 17-segment score derived from a 20-segment score for myocardial perfusion SPECT interpretation. J Nucl Cardiol 11: 414-423, 2004.

18. Hachamovitch R, Hayes SW, Friedman JD, Cohen I and Berman DS: Comparison of the short-term survival benefit associated with revascularization compared with medical therapy in patients with no prior coronary artery disease undergoing stress myocardial perfusion single photon emission computed tomography. Circulation 107: 2900-2907, 2003.

19. Smith SC Jr, Dove JT, Jacobs AK, et al; American College of Cardiology; American Heart Association Task Force on Practice Guidelines. Committee to Revise the 1993 Guidelines for Percutaneous Transluminal Coronary Angioplasty: ACC/AHA guidelines of percutaneous coronary interventions(revision of the 1993 PTCA guidelines) - executive summary. A report of the American College of Cardiology/American Heart Association Task Force on Practice Guidelines (committee to revise the 1993 guidelines for percutanous transluminal coronary angioplasty). J Am Coll Cardiol 37: 2215-2239, 2001.

20. Berman DS, Hachamovitch R, Shaw LJ, et al: Roles of nuclear cardiology, cardiac computed tomography, and cardiac magnetic resonance: Noninvasive risk stratification and a conceptual framework for the selection of noninvasive imaging tests in patients with known or suspected coronary artery disease. J Nucl Med 47: 1107-1118, 2006

21. Rispler S, Keidar Z, Ghersin E, et al: Integrated single-photon emission computed tomography and computed tomography coronary angiography for the assessment of hemodynamically significant coronary artery lesions. J Am Coll Cardiol 49: 1059-1067, 2007

22. Namdar M, Hany TF, Koepfli P, et al: Integrated PET/CT for the assessment of coronary artery disease: a feasibility study. J Nucl Med 46: 930-935, 2005 